\title{
What have nephrologists and cardiologists learnt from each other?
}

\author{
Eberhard Ritz and Andrew Remppis
}

Recently, even a modest degree of impaired kidney function (i.e. low estimated glomerular filtration rate or albuminuria) has been recognized as a powerful cardiovascular risk factor, with a predictive value comparable to that of the classical cardiovascular risk factors. This insight has led to the revision of past recommendations and the publication of remarkable joint statements by cardiology and nephrology experts (Sarnak MJ et al. [2003] Hypertension 42: 1050-1065) proposing that evaluation of renal function should be part and parcel of the work-up of patients with cardiovascular disease (Brosius FC III et al. [2006] Circulation 114: 1083-1087).

In the past, patients with chronic kidney disease were less likely to receive treatment (e.g. aspirin, $\beta$-blockers or statins) or undergo percutaneous coronary intervention for cardiovascular disease than were those without renal disease. Some risks of treatment, such as bleeding and technical complications, are certainly more frequent in renal patients, but, overall, cardiologists should treat renal patients with the same vigor as nonrenal patients.

Unfortunately, the optimal cardiological management of patients with end-stage renal disease has yet to be definitively established. Major impairment of renal function, particularly in a patient with diabetes, used to be considered a strong contraindication to cardiological intervention. Although things have changed dramatically since then, the management of cardiorenal disease, particularly in patients on dialysis, is still hampered by a lack of controlled prospective evidence. The unusually complex pathophysiology of cardiovascular disease in patients with renal disease is easily illustrated: 10 years ago, which cardiologist (or even nephrologist) would have considered serum phosphate or vitamin $D$ levels to be legitimate cardiovascular risk factors?

Clearly, cardiologists have learnt a lot from nephrologists. But what have nephrologists learnt from cardiologists? In a groundbreaking
.... it is time.... to motivate cardiologists to think more 'renal' and nephrologists to think more "cardiac"

E Ritz is Professor Emeritus in the Department of Internal Medicine and $A$ Remppis is a Cardiologist at Ruperto Carola University, Heidelberg, Germany.

\section{Competing interests}

The authors declared no competing interests.

www.nature.com/clinicalpractice doi:10.1038/ncpneph1001 observation, the Seattle group (Lindner A et al. [1974] N Engl J Med 290: 697-701) ascribed the excessive rate of cardiac death among patients on dialysis to myocardial infarction. Nephrologists have also learnt from cardiological research that not all coronary plaques are created equal, that unstable plaques can easily escape detection and that calcified plaques are not quiescent 'tombstones'; indeed, coronary calcification has turned out to be a strong predictor of cardiac events in renal patients.

Although the rate of myocardial infarction in patients with renal disease is much higher than in the general population, it has become apparent that the most frequent causes of cardiac death in the setting of renal disease are cardiac arrest and heart failure. The use of more sophisticated techniques has revealed that cardiomyopathy has a major role in the cardiac death of renal patients and that the pathogenesis of this condition is very complex. Cardiological research has also shown that diastolic malfunction contributes to the risk of pulmonary edema, on the one hand, and to the risk of hypotension during volume subtraction by ultrafiltration, on the other hand.

Furthermore, nephrologists have learnt from cardiology the importance of $\beta$-blockade, particularly in the context of excessive activation of the sympathetic nervous system and impaired breakdown of circulating catecholamines by lack of renalase. Finally, nephrologists have benefited from post-hoc analyses of large interventional cardiology trials, which have shown that renal patients profit enormously from interventions such as statins and blockers of the renin-angiotensin-aldosterone system, especially during the early stages of chronic kidney disease.

This incomplete list of the benefits obtained by exchanging ideas and information between cardiologists and nephrologists shows that it is time to make departmental barriers more permeable and to motivate cardiologists to think more 'renal' and nephrologists to think more 'cardiac'. United we are strong. 